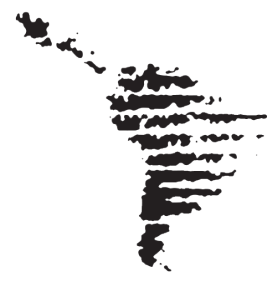

\title{
La accesibilidad de las TIC en Costa Rica: Un cambio disruptivo en la mente de la sociedad costarricense
}

\section{Accessibility to ICTs in Costa Rica: A Disruptive Change in the Mind of the Costa Rican Society}

\section{A acessibilidade das TIC em Costa Rica: A mudança disruptiva nas mentes da sociedade costarriquenha}

Darío Ríos Navarro

\section{Resumen}

El presente trabajo aborda la definición del concepto de accesibilidad universal, considerando datos del estado actual de la accesibilidad en Costa Rica, especialmente en el área de la tecnología. Tiene como objetivo concientizar y orientar al público lector, indicando algunos de los mitos que se han dado dentro de la definición de accesibilidad, tomando en cuenta diferentes perspectivas de otros estudios y ejemplificando situaciones relacionadas con la accesibilidad web, principalmente. Se analizan los datos generados mediante la INEC, sobre la población con discapacidad presente en Costa Rica, mediante información estadística, suministrada por los últimos censos realizados durante las décadas del presente siglo XXI, considerando la importancia de mejorar las TIC para las diferentes personas que presentan algún tipo de discapacidad, ocasionadas por naturaleza o por el tiempo. Realiza una revisión de diferentes proyectos que,

1 Graduado en Informática y Administración de Empresas en la Universidad Nacional, Costa Rica. Máster en Administración de proyectos por la Universidad para la cooperación Internacional (UCI). Académico de la Universidad Nacional y la Universidad Estatal a Distancia, Costa Rica. 
actualmente, se están fomentando en las TIC y que son avances importantes para mejorar las condiciones de las personas con discapacidad. Fomenta la necesidad inherente de trabajar en el desarrollo de las TIC en Costa Rica desde diferentes marcos. En el político y legal, identifica la necesidad de ampliar las normas que fomentan las leyes a favor de las discapacidades en el país. En conclusión, analiza que Costa Rica podría convertirse en uno de los primeros países en Centroamérica y el Caribe, que cubra las necesidades de las personas con discapacidad, mediante el fortalecimiento y el reconocimiento de las TIC que dan valor agregado para construir una accesibilidad universal.

Palabras clave: TIC, discapacidad, accesibilidad web y universal, Costa Rica.

\begin{abstract}
The present paper analyzes the definition of the concept of universal accessibility, considering the current state of accessibility in Costa Rica, especially in the field of technology. It aims to raise awareness and provide guidance to the public reader, pointing out some of the myths present within the definition of accessibility, taking into account different perspectives from other studies, and exemplifying situations related to web accessibility, mainly. We analyze the data generated by the National Statistics and Census Institute (INEC) on the population with disabilities in Costa Rica. This analysis is done through statistical information provided by the past censuses carried out during the decades of the present century, considering the importance of improving ICTs for people who have some type of disability, caused by nature or by time. The paper reviews different projects that, at present, are being promoted in ICTs and are considered important advances to improve the conditions of people with disabilities. It promotes the inherent need to work on the development of ICTs in Costa Rica from different frameworks. In the political and legal frameworks, it identifies the need to extend the rules promoting the laws in favor of disability in the country. In conclusion, the article analyzes that Costa Rica could become one of the first countries in Central America and the Caribbean covering the needs of people with disabilities, through the strengthening and recognition of ICTs that give added value to build a universal accessibility.
\end{abstract}

Keywords: ICTs, disability, web and universal accessibility, Costa Rica.

\title{
Resumo
}

Este artigo aborda a definição do conceito de acessibilidade universal, considerando dados do estado atual da acessibilidade em Costa Rica, especialmente na área de tecnologia. O objetivo é sensibilizar e educar o público, indicando alguns dos mitos que abarcam a definição de acessibilidade, tendo em conta diferentes perspectivas de outros estudos e exemplos de situações relacionadas principalmente com a acessibilidade web. 
Os dados sobre a população costarriquenha com incapacidade gerados pelo INEC são analisados, por meio de informações estatísticas fornecidas pelos censos recentes, realizados durante as décadas deste século XXI, considerando a importância de melhorar as TIC para as diferentes pessoas que apresentam algum tipo de incapacidade causada por questões naturais ou pelo tempo. Realiza-se uma revisão de diferentes projetos que atualmente estão sendo promovidos nas TIC e são importantes avanços na melhoria das condições das pessoas com deficiência. Promove a necessidade inerente de trabalhar no desenvolvimento das TIC na Costa Rica a partir de diferentes quadros. No campo político e legal, identifica a necessidade de ampliar as regras que incentivam as leis em favor das incapacidades no país. Em conclusão, analisa que a Costa Rica poderia tornar-se num dos primeiros países da América Central e do Caribe, que atende às necessidades de pessoas com incapacidade, por meio do fortalecimento e reconhecimento das TIC que dão valor acrescentado para construir uma acessibilidade universal.

Palavras-chave: TIC, incapacidade, web e acessibilidade universal, Costa Rica.

\section{Introducción}

El presente artículo pone en evidencia las necesidades de fomentar la accesibilidad en materia de las tecnologías de información, como un proceso para crear conciencia en el tema de accesibilidad universal en Costa Rica. Si bien es cierto que se ha empezado a brindar una mayor oportunidad de desarrollo en este tema, aún el hablar de la accesibilidad sigue envuelto en mitos que del todo no se han podido superar, así como de diferentes retos que muchas organizaciones no están completamente dispuestas a enfrentar, por diferentes razones que representan una prioridad. Se busca hacer un análisis sobre el estado actual que se presenta a nivel nacional, desde el punto de vista legal, político y social relacionado con las personas con discapacidad. Así mismo, identificar las expectativas que se tienen para el futuro sobre este tema, mediante un análisis de la situación y verificando cómo Costa Rica puede llegar a convertirse en un país promotor en el proceso de accesibilidad de las tecnologías de la información y la comunicación (TIC) para Centroamérica y el Caribe.

\section{Accesibilidad universal}

Para empezar, es importante mencionar el concepto propio de accesibilidad, ya que esta definición puede enfocarse desde diversas perspectivas. Tanto en libros, como en la internet, se encontrarán diferentes términos y razones que 
hacen alusión a este tema, y que en su mayoría pueden ser definiciones correctas. Sin embargo, para justificar el término, se hará referencia a la definición de accesibilidad universal. Según un artículo publicado por el Ministerio de Trabajo y Asuntos Sociales de España, y dirigido por un equipo de investigación de la Universidad Carlos III de Madrid, se define así:

La accesibilidad universal se presenta como una condición ineludible para el ejercicio de los derechos -en igualdad de oportunidades- por todos los individuos, y para, en último término, conseguir el logro de la igual dignidad humana, del libre desarrollo de la personalidad, de todas las personas. En ese sentido, no puede ser vista como una cuestión sujeta a la voluntad política, facultativa y graciable, o como una técnica para la rehabilitación de ciertas personas. (Roig, 2013, p. 6.).

Partiendo de esta definición, se especifica el concepto y a su vez se muestra un panorama mucho más amplio de todo lo que implica este término. Y es que el proceso de accesibilidad busca crear oportunidades para todas las personas, basándose en conceptos de universalidad y bienestar social. Esta definición hace un hincapié en que la accesibilidad es un proceso incremental que busca quebrantar algunas barreras a las cuales las personas se han acostumbrado, y las ven como algo muy normal. Por eso, el título de este artículo busca dotar a las personas de ese cambio disruptivo que necesitamos para poder entender que la accesibilidad es un fin común para todas las personas. Y no únicamente a nivel país, sino también a nivel mundial como un esfuerzo en conjunto.

Normalmente, y recabando un poco más de información, podemos encontrar de diversas fuentes, el término accesibilidad web, término en el cual vamos a entrar más a fondo y donde podemos hacer referencia a la definición brindada por Dialnet de la Universidad de la Rioja en España que dice lo siguiente: "Se conoce por accesibilidad web, la posibilidad de que cualquier persona, independientemente de sus capacidades personales y de las características técnicas del equipamiento utilizado, tenga acceso a toda la información y funcionalidades de un sitio web" (Dialnet, s. f., párr. 1).

De todas las definiciones, esta parece una de las más apropiadas, porque se enfoca en detallar y fortalecer el concepto de accesibilidad universal. Y trata de valorar la necesidad de verificar que la accesibilidad es la posibilidad de que cualquier persona, independientemente de sus capacidades, pueda tener acceso 
a la información que brinda la internet. Esto relaciona ambas definiciones en el proceso de crear igualdad de oportunidades. En el ámbito de este tema de accesibilidad, y desde el punto de vista de la informática, me parece conveniente referirme más al término de accesibilidad en las TIC, el cual se retomará más adelante, para analizar el porqué de su importancia y cómo engloba aspectos más allá de la accesibilidad web como tal.

\section{Mitos de la accesibilidad web}

Algunos de los llamados mitos que existen en la accesibilidad -unos conocidos y otros no tan conocidos- se exponen al lector en este artículo. El propósito es analizar la problemática y cómo o qué clase de errores se están cometiendo, los que afectan de manera diferente la situación de personas que necesitan de la accesibilidad. Se considera qué se podría estar haciendo o no al respecto. Muchos de los mitos que se presentan son considerados no solo en el país, sino también en muchos otros lugares que se han ido involucrando en el proceso. Preocupa que una población importante aún tenga una mala percepción de lo que está ocurriendo.

\section{Mito 1: La accesibilidad web es un proceso que busca ayudar a las personas con alguna discapacidad}

Si bien es cierto que esa es una de las finalidades de este tópico, tomando en cuenta la accesibilidad universal, se puede decir que aún no se está observando el panorama completo. Congruentemente, el fin común es ayudar a personas con discapacidad, de las cuales las más reconocidas son las personas con ceguera, con sordera o con alguna dificultad motora. Se han hecho avances importantes en el desarrollo de espacios físicos, pero aún falta mejorar procesos en la parte de accesibilidad web y las TIC. Y no solamente se trabaja para personas con algún tipo de discapacidad como las mencionadas previamente, como se ejemplifica a continuación.

Uno de los problemas de accesibilidad que se ha estado trabajando son los medios para llegar a la población indígena del país. Las personas de estos grupos autóctonos, aunque algunas no cuentan con ningún tipo de discapacidad, por el lugar en donde se encuentran ubicadas sus comunidades y por dificultades técnicas que aún existen en relación a la tecnología (e inclusive a la resistencia a los cambios tecnológicos, pero eso ya corresponde a otro tema) del todo no tiene acceso a ciertos servicios o solo limitado. Esto representa un claro problema de accesibilidad. 
La FONATEL (Fondo Nacional de Telecomunicaciones) recientemente presentó, en un congreso de accesibilidad de la UNESCO, el proyecto Acceso universal, servicio universal y solidaridad (SUTEL, s. f.). En este destaca un análisis de cómo se está trabajando con varias poblaciones indígenas de nuestro país, a lo largo del territorio nacional, de tal manera que se pueda mejorar el acceso al internet en hogares, comunidades y espacios públicos. Esto, con el fin de brindar mejores condiciones para la población de estas zonas. Es un avance de suma importancia como proceso inicial para llegar a conectar a las personas a los medios tecnológicos, lo cual después llevará a hondar en que necesitamos mejorar los aspectos de accesibilidad web para colaborar en los medios de educación y socialización de este amplio sector. En la figura 1, se muestra una imagen con las diferentes etapas del reglamento propuesto por dicha entidad, que afectan todas las zonas tanto urbanas como rurales. Es un proyecto de mucha visión que pretende solucionar muchas limitantes, sobre todo a nivel de comunicaciones. Nótese que el proceso no está hecho con el fin de solo ayudar a personas con algún tipo de discapacidad; por el contrario, se busca fomentar el acceso a las tecnologías, para todas las personas, supliendo con acceso al internet y a la tecnología en igualdad de condiciones.

\section{Tabla 1}

Cinco fases consideradas en el reglamento de acceso universal

\section{Fases del proyecto FONATEL}

\section{Comunidades conectadas}

Servicio de internet y telefonía en zonas alejadas.34 proyectos ejecutados. 1 millón de personas beneficiadas. Inversión: \$150 dólares.

3. Centros públicos conectados. Computadoras para escuelas, colegios, CECIS, Cencinai y centros de salud. Inversión: \$20 millones

5. Red de banda ancha solidaria. Soporte con banda ancha a zonas rurales (En formulación en el 2017)

\section{Hogares conectados}

Internet fijo, computadoras y contenidos digitales a familias de escasos recursos. 140 mil familias beneficiadas. Inversión: $\$ 120$ millones.

4. Espacios públicos conectados. Red nacional de internet gratis en parques. 240 zonas de conexión. 177 comunidades beneficiadas. Inversión $\$ 20$ millones.

Nota: Elaborada con base en la presentación de FONATEL (2016). 
En la figura 1, se muestra que actualmente, para el sector de la población indígena, se están considerando las dos primeras fases presentadas por el reglamento, identificadas como comunidades y hogares conectados, que como se especifica en tabla 1, buscan brindar desarrollo en equipos e internet a esta importante población de nuestro país. Dadas las condiciones que se presentan en estos sectores, ya sea por el difícil acceso y cobertura de red que existe, se puede decir que es un completo beneficio para las personas de los centros y áreas indígenas, porque al tener un mayor acceso a la tecnología, también tienen mejores vías para comunicarse y para desarrollar su educación. Por ejemplo, para las amas de casa, la niñez y jóvenes adolescentes, se brindan beneficios con el fin de solventar los problemas en la educación, donde estos grupos quieren realizar sus estudios y, por las dificultades de la zona, a veces se les hace difícil movilizarse a los centros de estudio, ya que estos se encuentran a muchos kilómetros del lugar donde residen. Se facilita la comunicación para fines como, por ejemplo, cuando es necesario acceder a servicios de salud, ya sea por situaciones anómalas o emergencias de alto riesgo que se produzcan en el lugar.

\begin{tabular}{|l|l|}
\hline \multicolumn{2}{|c|}{ Fases del proyecto FONATEL } \\
\hline \multicolumn{1}{|c|}{ 1. Comunidades conectadas } & \multicolumn{1}{|c|}{ 2. Hogares conectados } \\
\hline Servicio de internet y telefonía en & Internet fijo, computadoras y contenidos \\
zonas alejadas.34 proyectos ejecutados. & digitales a familias de escasos recursos. \\
1 millón de personas beneficiadas. & 140 mil familias beneficiadas. Inversión: \\
Inversión: \$150 dólares. & $\$ 120$ millones. \\
\hline
\end{tabular}

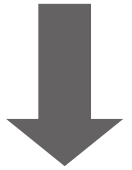

Población indígena

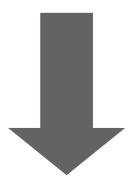

Territorios indígenas

Figura 1. Las dos fases del proyecto FONATEL que actualmente benefician a nuestra población indígena. Basado en la presentación de FONATEL (2016).

Por otro lado, se sabe que algunas discapacidades son congénitas o por algún tipo de accidente. Pero también, como su nombre lo indica, existen aquellas discapacidades que se presentan de manera temporal sobre una persona. La AGESIC (Agencia para el Desarrollo del Gobierno de Gestión Electrónica y la Sociedad de la Información y del Conocimiento) en Venezuela, 
es un organismo que organiza los procesos de gobierno digital en ese país, y en su capítulo III, desarrollado en el 2012, define algunas discapacidades temporales, que se citan a continuación:

Las personas mayores que han visto disminuidas sus habilidad a consecuencia de la edad, las personas con bajo nivel de alfabetización o no habla el idioma, las personas con conexiones de bajo ancho de banda o la utilización de tecnologías más antiguas, a los usuarios nuevos y poco frecuentes, a usuarios que operen en contextos muy diferentes de los ideales, que no sean capaces de ver u oír, leer o entender texto, usar un teclado o ratón, o hablar con claridad, a usuarios que puede ser que no tengan discapacidades pero tengan los ojos ocupados o las manos ocupadas, ambiente ruidoso o necesidad de silencio, ancho de banda estrecho o tamaño de pantalla o colores limitados. (AGESIC, 2012, p. 125)

Algunos de los aspectos a los que se hace referencia en el capítulo como discapacidades temporales, pueden ser cuestionables dadas ciertas circunstancias descritas, como tener las manos ocupadas. Sin embargo, para citar un ejemplo muy puntual sobre personas que operen en circunstancias distintas de las ideales, es conveniente tomar en consideración el siguiente ejemplo. Un joven estudiante llamado Luis, el cual no cuenta con ningún tipo de discapacidad, está realizando un trabajo de investigación en una biblioteca. Él encuentra en internet una referencia de un vídeo que parece aclarar ciertas dudas, que tiene en relación con un proyecto que está efectuando. Él quiere ver el video, pero debido al lugar donde se encuentra ubicado, se le ha dicho que debe mantenerse en silencio. Ese día, Luis ha dejado los audífonos en su hogar, por lo cual no puede hacer uso de esa ayuda técnica. Intenta poner el video con poco volumen, pero hay demasiado silencio; lo cual puede perturbar a otras personas que se encuentran cerca. Igualmente, el video no se escucha tan bien para identificar lo que se está diciendo.

Una solución viable, sería pensar: muy sencillo, puede pasarse a un lugar donde sí pueda reproducir el vídeo, o salir de la biblioteca. Posiblemente den con una solución para este problema tan sencillo, pero hay que recordar que la accesibilidad universal habla de igualdad de oportunidades. La solución anterior realiza una exclusión del lugar, característica que no facilita ni soluciona adecuadamente un problema de este tipo desde el punto de vista de la accesibilidad. La solución que podría haber solventado las necesidades de Luis, es el que video contará con subtítulos, ya que, aunque no tuviera los audífonos, podría haber 
entendido lo que estaba ocurriendo para resolver su cuestionamiento en la investigación. Esto, por citar un breve ejemplo de una situación de discapacidad temporal y porque no se debe creer, bajo ninguna circunstancia, que solamente con desarrollar accesibilidad se está favoreciendo a solo cierto grupo de personas con discapacidades de audición o ceguera.

Las llamadas discapacidades, van más allá de los aspectos físicos de los cuales se habla continuamente. Se han realizado estudios y análisis de la población, donde se acostumbra entender como problemas físicos o sensoriales en una persona de forma permanente, lo cual no siempre es cierto, como se ha desmentido en este mito. Así mismo la orientación que debe darse a la accesibilidad en diferentes términos se irá solucionando paulatinamente, conforme aboquemos en desarrollar las necesidades de accesibilidad que diferentes personas tienen en diversos tiempos.

\section{Mito 2: Contamos con la ley 7600 que cubre los aspectos necesarios para las personas con algún tipo de discapacidad}

En el desarrollo de este artículo, no se busca efectuar un análisis exhaustivo de la ley, porque el autor no es experto en leyes; sin embargo, se aclara que se ha tomado el tiempo de leerla y de denotar, cómo está compuesta y qué permite esta ley. Para ello, se identifica que aún se encuentra en un proceso inicial de definir, con mayor detalle, artículos relacionados con el área tecnológica. La ley generaliza respecto al uso de ayudas técnicas y servicios de apoyo. El artículo 50 de la ley dice:

Artículo 50- Información accesible

Las instituciones públicas y privadas deberán garantizar que la información dirigida al público sea accesible a todas las personas, según sus necesidades particulares. (Ley 7600, p. 13)

La intención que se busca a través de la información accesible es la meta a la cual deben dirigirse todos los esfuerzos para una accesibilidad universal, pero quedan muchos detalles que evaluar para alcanzar este proceso, considerando la tecnología como medio para llegar a ese objetivo. Esto no es un problema que se le está achacando al gobierno por el desarrollo de esta ley y no considerar entre todos sus aspectos, la parte de las TIC. Por el contrario, es una oportunidad para mejorar los reglamentos, normas y políticas que, basados en esta ley, puedan fortalecer y dar una mejor orientación a la accesibilidad en el área de las tecnologías. 


\section{Mito 3: El término de "sensibilización” en accesibilidad}

Este mito es uno de los más conocidos y tiene que hablar con una situación generalizada sobre este tema de accesibilidad universal. La palabra sensibilizar según la RAE (Real Academia Española) corresponde a: "Hacer sensible algo o a alguien"(RAE, 2016). La mayoría de personas que están más familiarizadas con la accesibilidad saben que sensibilizar no es una palabra adecuada para referirse al proceso. Basado en el diálogo de accesibilidad universal, que se consideró al inicio, no se trata de pensar que hay que ser sensible con una persona para que esta persona se sienta bien y las demás personas se sientan bien consigo mismas. Está tradicional forma de pensar, en Costa Rica se podría llamar el síndrome del "pobrecito". En lugar de eso, se trata de involucrar en el desarrollo de las actividades a una persona con algún tipo de discapacidad, fortaleciendo su interacción y dándole el mismo grado de igualdad que otra persona. Por tanto, al "sensibilizarlo" o ser parte del síndrome descrito anteriormente, lo que se está creando es una imagen errónea hacia las demás personas, e inclusive hacia la persona con discapacidad, que es una persona que necesita que sientan algún tipo de compasión, lo cual es verdaderamente incorrecto.

La forma más correcta de referirse a un proceso de este tipo es usar la palabra concientizar. Volviendo a la RAE, la definición dice: "Hacer que alguien sea consciente de algo" o "Adquirir conciencia de algo" (RAE (2), 2016), donde se habla de crear conciencia en las personas con discapacidad o sin esta para hacerles ver la importancia de este asunto y cómo, de una forma u otra, esto concierne a toda la población.

\section{Mito 4: Hacer un sitio web es complicado y caro}

Este mito se ha escuchado y leído muchas veces, y normalmente es un tema de discusión. Pero, realmente, se puede analizar con detenimiento, ¿acaso la accesibilidad no busca ser un bien para todos y todas que valdría la pena realizar? ¿No se invierte en construir sitios web con determinadas características y que tengan un adecuado rango en los buscadores de internet para que aparezcan de primeros? Son muchas las necesidades que se presentan para lograr una ventaja competitiva del negocio, pero a veces no se toma en cuenta que esos aspectos que se consideran de alto coste pueden brindar una mejor rentabilidad, al integrar a todo tipo de personas; y, a su vez, estamos contribuyendo con una labor de responsabilidad social. En un artículo publicado en el periódico la nación, el 20 de octubre del 2015, Lecuona hace importantes aportes relacionados a este mito. El expresa: 
A veces se tiene la idea de que hacer las cosas accesibles es muy caro, y eso ocurre porque se trata de modificar lo ya diseñado. Pero si diseño de entrada pensando en que las cosas sean más accesibles, no es más caro. (AFP, 2015, párr. 13)

Si pensamos en el desarrollo de sitios y aplicaciones web, considerando en primera instancia la accesibilidad, los costos serían mucho menores que hacer un reproceso de un sitio o aplicación. Pero muchas veces se dejan estos aspectos de lado, omitiendo la importancia a futuro que realmente se merece este tipo de actividades, que eventualmente se podrían volver más costosas por una mala planificación inicial.

Por otro lado, como se ha comentado anteriormente, hay diferentes tipos de discapacidades que se deben trabajar, y que pueden volver complicada la implementación, lo cierto es que también se cuenta con los medios para lograr que muchos de los objetivos se lleguen a cumplir. Lecuona y Guala, en ese mismo artículo mencionan:

"Hay muchas limitaciones. A veces se piensa que hacer accesible un siti o web es algo muy complejo y no tiene por qué serlo", señaló Guala, en tanto Lecuona enfatizó que sería importante que al momento de desarrollar una página pública en internet se considere que "su contenido pueda ser utilizado por los diferentes niveles de usuarios (videntes, no videntes, o portadores de otras discapacidades)". (AFP, 2015, párr. 10)

Por medio de la W3C (World Wide Web Consortium), que es una de las organizaciones más importantes en la internet, existen las llamadas para el contenido web, también conocidas como WCAG. Dichas pautas de accesibilidad de la web dan una explicación de cómo realizar sitios web accesibles partiendo desde el contenido en HTML (HyperText Markup Language), el lenguaje comúnmente utilizado por los navegadores desde hace muchos años (Mitchell, s. f.). Estas pautas brindan una explicación de cómo llegar a obtener un grado de accesibilidad mediante tres niveles: A, doble A y Triple A. Normalmente, se requiere trabajo para realizar un sitio completamente accesible ( $Y$ normalmente no se puede hablar de sitios o aplicaciones que tengan un cien por ciento de accesibilidad), pero lograr un nivel A de accesibilidad no tiene por qué llegar a ser costoso o complicado. La visión de ese primer nivel de las pautas mencionadas es que un sitio o aplicación cuente con características que normalmente todas las 
aplicaciones deberían tener como un estándar de desarrollo para las personas que ingresan a determinado sistema. Si indagamos en los otros niveles, es posible que se tenga que invertir un poco más para lograr que se cumplan ciertos criterios para un nivel AA o nivel AAA. Pero como propósito incipiente, de manera objetiva hay que iniciar con lo básico, para ir pasando cada uno de los niveles que se requieren para alcanzar un porcentaje de accesibilidad alto.

Mediante este aporte, se han considerado algunos de los mitos más comunes de la accesibilidad web y la accesibilidad en general, aunque hay muchos más que se pueden desglosar. Normalmente, todos relacionados con costo, dificultad e inclusive con estética de los sitios web.

\section{Personas con discapacidad en Costa Rica}

En un estudio realizado en el año 2004 por el Ministerio de Salud, se detalló que mediante el censo del año 2000 en Costa Rica, se obtuvo información presentada en la figura 3 y brindada por dicho ministerio, donde indica que para ese momento, la población de Costa Rica correspondía a 3810179 personas en su totalidad. De esa población, las personas con discapacidad representan un total de 203731 entre hombres y mujeres. Esto representaría aproximadamente el 5,34 por ciento de la población total.

Este mismo estudio reveló que hay una mayor población masculina con discapacidades. Sin embargo, en relación con la población femenina para esa época, no resultaba haber una gran diferencia.

\section{Tabla 2}

Distribución de la población con discapacidad en Costa Rica por género, según característica de población para el año 2000

(Valores absolutos)

\begin{tabular}{lrrr}
\multicolumn{1}{c}{ Población } & \multicolumn{1}{c}{ Total } & Hombres & \multicolumn{1}{c}{ Mujeres } \\
\hline Costa Rica & 3810179 & 1902614 & 1907565 \\
Población con discapacidad & 203731 & 105271 & 98460 \\
\hline
\end{tabular}

Nota: Basado en los datos del MINSA (2004). 
Por medio del estudio, se definieron 6 grupos para describir las necesidades de las personas en: ciegas, sordas, con algún tipo de retardo mental, parálisis o amputación, algún tipo de trastorno mental y otros. En la figura 4, que presenta el cuadro 2, se establecen dichas características también agrupadas por género. En este caso, para el año 2000, se diferenciaban mayor cantidad de personas con algún tipo de discapacidad según la parte de ceguera parcial o total; y en otros. Hay que considerar también, que el tipo de discapacidad se puede ver influenciada por factores como el tiempo, que para dicho estudio se concluyó que en la parte de ceguera y otros, corresponde un porcentaje alto para las personas de los grupos etarios mayores (MINSA, 2004). También indica que:

Sin embargo, existen otras discapacidades no registradas que alcanzan la cifra de 54913 personas. En suma, entre la ceguera parcial o total y otras discapacidades no clasificadas por el Censo Nacional, se encuentra un valor acumulado de un 3,09\%. (MINSA, 2004, pág. 19)

Esto demuestra que para la época que se realizó el estudio, este porcentaje de la población, tenía un valor lo suficientemente alto sobre la población total con discapacidad. Aunque lamentablemente, el estudio no explica completamente, la categoría considerada como todos, para saber realmente a qué clase de discapacidades se refiere.

Tabla 3

Distribución de la población por tipo de discapacidad, según género en Costa Rica, para el año 2000

\begin{tabular}{ccccccc}
\hline Población & $\begin{array}{c}\text { Ceguera } \\
\text { parcial o } \\
\text { total }\end{array}$ & $\begin{array}{c}\text { Sordera } \\
\text { parcial o } \\
\text { total }\end{array}$ & $\begin{array}{c}\text { Retardo } \\
\text { mental }\end{array}$ & $\begin{array}{c}\text { Parálisis o } \\
\text { amputación }\end{array}$ & $\begin{array}{c}\text { Transtorno } \\
\text { mental }\end{array}$ & Otro \\
\hline Costa Rica & 62556 & 26235 & 18880 & 28119 & 12729 & 55212 \\
Hombres & 32784 & 15178 & 10899 & 15779 & 7288 & 23343 \\
Mujeres & 29772 & 11057 & 7981 & 12340 & 5441 & 31849 \\
\hline
\end{tabular}

Nota: Basado en los datos del MINSA (2004). 
En un estudio más reciente, suministrado también por INEC, que toma como base el censo del 2011, se muestra lo siguiente:

\section{Tabla 4}

Distribución de la población con discapacidad en Costa Rica por género, por características de población, según el censo del año 2000

\begin{tabular}{lrrr}
\multicolumn{1}{c}{ Población } & \multicolumn{1}{c}{ Total } & Hombres & \multicolumn{1}{c}{ Mujeres } \\
\hline Costa Rica & 4301712 & 2106063 & 2195649 \\
Población con discapacidad & 452849 & 218395 & 234454 \\
\hline
\end{tabular}

Nota: Realizada por el autor tomando de referencia los datos suministrados del censo del 2011 publicados por INEC (2011).

Como se puede notar, la población de nuestro país, del año 2000 al año 2011, aumentó considerablemente para un $12 \%$ más con respecto al censo anterior. De igual forma, predomina el género femenino, sin embargo, podemos asimilar que hay un incremento sustancial en las personas con discapacidad de este género, a diferencia del año 2000. Los resultados de la tabla 1 se cruzan con los resultados de la tabla 2, y podrá notarse que las cantidades totales de personas con discapacidad difieren entre ellas. Esto se debe a que en el reporte del INEC sobre la población, hace una salvedad indicando que pueden haber personas que tengan varios tipos de discapacidad (por ejemplo, una persona puede tener problemas para ver aún con lentes puestos y también tenga un problema del tipo mental), lo cual denota nuevamente que no solamente trabajamos para personas con una sola discapacidad, sino que se debe considerar que el acceso a las TIC debe favorecer, de la mejor manera, a todas las discapacidades que se manifiesten sobre una persona. La tabla 2, como se explicó a grandes rasgos anteriormente, cruza las personas con discapacidad según una categoría propia realizada, e incluye a las personas sin ningún tipo de discapacidad. Si tomamos la diferencia de la población tanto de hombres como de mujeres que no tienen ningún tipo de discapacidad y la población total, se obtendrán los resultados de población con discapacidad de la tabla 1. Sin embargo, si se suman todas las columnas que categorizan las distintas discapacidades, en el caso de los hombres; se obtendrá un total de 287214 personas con discapacidad y en el caso de las mujeres, sería un total de 315 398. Dichos datos no coinciden con la tabla 1 por lo señalado. 


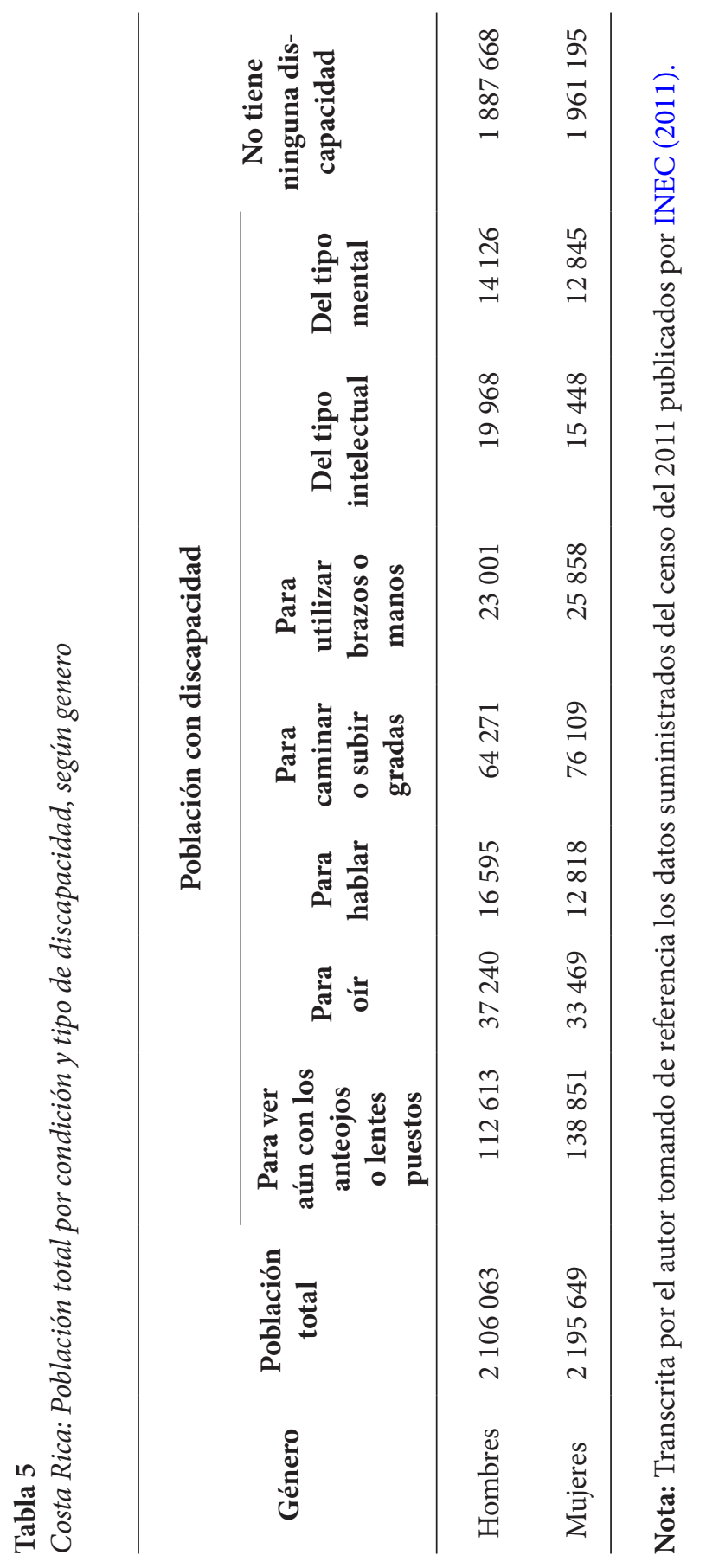


La expectativa que se tiene supone que, con el paso de los años, aumente la natalidad y haya una disminución por mortalidad, lo cual daría como resultado que, en Costa Rica, exista una población con discapacidad mayor (generada principalmente por el aumento de la edad). Por esto se debe procurar contar con las medidas necesarias para darle adecuados medios para tener acceso a espacios físicos, como se ha ido implementando hasta ahora, pero también, en el uso de las tecnologías de información.

\section{Avances en proyectos de accesibilidad en Costa Rica}

De vuelta al estudio suministrado por el ministerio de Salud en el año 2004, se analiza lo siguiente:

Por su parte, el derecho a la información y a la comunicación, establecido en los artículos del 50 al 53 de la Ley 7600, en términos generales, ha tenido un muy lento progreso.

Sin embargo, las innovaciones que se han generado, tales como: servicio 137 de relevo telefónico, facturación de algunos servicios en braille, noticiarios de televisión con interpretación en LESCO, han sido trascendentes para la población con discapacidad. (MINSA, 2004, p. 69)

Para el año referenciado en dicho documento, se puede decir que se han realizado mejores avances en las actividades que incluyen a las TIC en Costa Rica, pero aún falta gran camino por recorrer. Es importante reconocer algunos de los proyectos que han tenido auge en mejorar y facilitar el desarrollo de las TIC para personas con discapacidad en nuestro país, entre las cuales se mencionan:

- El proyecto Periscope que impulsa Kolbi como proyecto innovador en el desarrollo de una canal web con programas televisivos especiales para personas sordas (Vargas, 2016).

- El proyecto POETA que es una iniciativa de la fundación Omar Dengo en conjunto con el Ministerio de Educación Pública. Uno de los aspectos más destacable de este proyecto, como menciona Molinas y Cuevas, es:

El proyecto Poeta de la FOD se sustenta en la certeza que las personas con discapacidad no cuentan con lugares donde aprender a utilizar las herramientas digitales y esta población tiene derechos y debe aprender 
a ser independiente y apoyar el desarrollo del país. Para la Fundación Omar Dengo, fue un reto asumir procesos de capacitación para personas (jóvenes en su mayoría) con discapacidad cognitiva. (Molina, p. 16)

Aunado a este comentario que hacen los autores, se trabaja fuertemente en la inclusión laboral de estas personas en ambientes donde haya una igualdad de condiciones.

- El Tecnológico de Costa Rica (TEC) realiza, una vez al año, la jornada de accesibilidad digital. La tercera jornada se celebró en octubre del año 2016, la cual contó con la participación de varios conferencistas, tanto nacionales como internacionales, donde se trabajaron varios temas en materia de accesibilidad por medio de las TIC. Entre los proyectos presentados por el TEC, se destacó el desarrollo de un avatar virtual que traduce las palabras a LESCO. Además, se están desarrollando sitios web que presenten características accesibles (mediante documentos y páginas accesibles) (Mora, 2016).

- FONATEL se ha involucrado en el proceso de desarrollar una mejor conectividad en Costa Rica, y espera realizarlo de manera total para el presente año 2017. El proyecto que se explicó anteriormente de hogares conectados es una iniciativa que busca reducir la brecha digital en nuestro país. Esto es un gran paso para el tema de telecomunicaciones. En datos suministrados por el financiero, se especifica que durante los años 2013 a 2014, hubo un incremento de suscripciones al internet de un $61 \%$, lo cual representa un importante valor, porque con el aumento de acceso a las tecnologías, también se influye en el desarrollo de software del país, consolidando soluciones para la "nube" y big data (gran almacenamiento de datos). También como se menciona en la noticia, se busca equilibrar el acceso a la tecnología para diferentes estratos sociales (Chacón, 2015).

Según un informe del año 2003, presentado por la Universidad de Costa Rica (UCR), los gobiernos expresaron su compromiso por construir una sociedad de la información centrada en las personas y orientada a un mejor desarrollo. En el 2011, se comenta en el artículo:

Se reconoce que, a pesar de los esfuerzos, sólo un $32 \%$ de la población mundial (Banco Mundial 2010) forma parte de esta sociedad, el resto está privado de los beneficios que ella provee. Muy probablemente, la 
gran mayoría de las personas con discapacidad están entre quienes tienen menores posibilidades de hacerlo. Esa estimación se fundamenta en el hecho de que la discapacidad está altamente vinculada a la pobreza y existe una alta correlación entre disponibilidad de medios económicos y acceso real a las TIC. (PROSIC - UCR, 2011, p. 18)

En la actualidad, se han sumado esfuerzos y se ha promovido la erradicación de la brecha digital existente, en las zonas del país de difícil acceso. Por ello, es posible que encontremos una disminución en los hechos destacados de este informe, producto de los proyectos actuales que se fomentan en Costa Rica, lo cual también erradica, hasta cierto punto, aspectos en el área de la tecnología para las personas sin acceso por sus condiciones económicas.

\section{Hacia una Costa Rica promotora de la accesibilidad en Centroamérica y el Caribe}

En una noticia publicada por Carreño, en el 2016, una página de noticias en México, se habla de las necesidades que se tienen que solventar en materia de accesibilidad para diferentes países de Centroamérica y América del Sur. Gloriana Monge realiza un comentario sobre cómo el plan nacional de desarrollo de telecomunicaciones, propuesto para los años 2015 - 2021 en Costa Rica, va a fomentar mejor calidad de vida por medio de las TIC. La vía para realizar dicha tarea se basa en tres pilares que son: la inclusión digital, el gobierno electrónico y la obtención de un sistema de economía digital. Una de las características más sobresalientes de ese plan consiste en que se busca integrar a los sitios web del Estado para lograr su accesibilidad. No se sabe hasta qué nivel se pretende llegar con esta propuesta, pero llama la atención que se trabajará para realizar reconocimientos y un premio anual de TIC accesible, a las organizaciones que participen en el proceso. Este debe ser un incentivo importante para lograr el objetivo de acercar tanto a empresas gubernamentales como no gubernamentales en el desarrollo de tecnologías accesibles. Pero se deben seguir focalizando esfuerzos en concienciar a las organizaciones del porqué se deben realizar sitios y aplicaciones web accesibles; y dar a conocer las herramientas tecnológicas, preferiblemente de software libre, que permitan mejorar las necesidades de diferentes tipos de discapacidades.

Además, Costa Rica puede involucrarse aún más en la promoción de turismo accesible. Aunque no es tema central en este documento, se puede decir que puede apoyarse de las TIC para estos fines. 
Durante el congreso de la UNESCO realizado en el año 2016 en Costa Rica, asistieron personas de diferentes países, tanto de Europa, América del Norte y Sur; y América Central. Estas se involucraron en el análisis de la situación de Latinoamérica y el Caribe, donde indicaron información por parte de la G3CIT, la cual informa que, en Centroamérica, hay un 33\% de ayuda financiera para empresas estatales y no gubernamentales en trabajos de soporte a la accesibilidad digital. Esto representa un porcentaje bajo, para los esfuerzos que se pueden estar realizando en el tema, pero dados los logros que se han presentado en diferentes proyectos que se manejan actualmente, especialmente en la labor de solventar la brecha digital, el siguiente paso que debe realizarse es dar a conocer y promover, en Costa Rica, una nación en búsqueda de igualdad de oportunidades, desarrollo personal y profesional para las personas, independientemente de sus condiciones físicas y mentales, e idealizar la promoción de software y hardware accesible. En el estudio que se promovió en el Congreso, también se habló de algunos datos en los cuales Costa Rica gratamente lidera. La CEPAL muestra los resultados obtenidos por América Latina y el Caribe en el uso de internet en personas con discapacidad, en un criterio de edad que va de los 25 años o más y por nivel de escolaridad. Costa Rica se posiciona en primer lugar, y esto se debe, en gran manera, a que, aunado a otros estudios, se ha trabajado fuertemente en mejorar la deserción escolar y el acceso a la tecnología.

Por otro lado, en ese mismo estudio, se puede identificar que si se toma la población completa de personas con discapacidad y sin discapacidad, divididas por sexo, se puede identificar que Costa Rica está como segundo país en brindar, a la población, las condiciones adecuadas para el uso del internet. En Antigua y Barbuda, que al parecer tiene mayor población que hace uso del internet según este análisis, sobresale el acceso de las personas con discapacidad del género femenino, pero a diferencia de Costa Rica (que se puede visualizar que el acceso al internet es muy equitativo entre ambos sexos), hay una disminución en el género masculino, respecto al uso de internet. Esto puede diagnosticar en este aspecto, que las situaciones de uso de internet, para Costa Rica, son bastantes buenas respecto a otros países.

Ciertamente, estos datos no son de obviar y se debe analizar que con las propuestas que ha hecho el país durante los últimos años y en relación con la información suministrada, podría tomarse beneficiosas medidas y ventajas para posicionar al país como uno de los primeros precursores en el acceso de la tecnologías de la información y la comunicación de Centroamérica, enfocado en aplicar esfuerzos en el desarrollo de las personas con discapacidad; para suministrar diferentes tipos de tecnologías accesibles que mejoren su interacción y uso de la internet. 


\section{Realizando un cambio disruptivo}

El proceso de cambio que se busca realizar en el tema de accesibilidad de las TIC está lleno de mitos e incertidumbres que se deben evaluar, mitigar y eliminar. Hay estrategias para desarrollar la accesibilidad en diferentes organizaciones, principalmente del Estado. Sin embargo, aún falta impulsar al sector público y privado en el desarrollo de estrategias de accesibilidad como un fin para aumentar su productividad y mejorar las condiciones de igualdad necesarias para alcanzar la accesibilidad universal.

La legislación no es lo suficientemente explícita respecto a los procesos de accesibilidad en materia de TIC. Es necesario formalizar políticas y normas adecuadas que permitan desarrollar el uso de las TIC en diferentes organizaciones. Sería de suma importancia que el Ministerio de Ciencia, Tecnología y Telecomunicaciones (MICITT), junto con otras organizaciones gubernamentales y no gubernamentales, tomen conciencia del proceso de formalizar el desarrollo de la tecnología para personas con discapacidad. La ley 7600 y la ley 9379 (ley más reciente, que se publicó en agosto del año pasado), abarcan diferentes aspectos de las necesidades de personas con discapacidad desde el punto de vista de espacios físicos y de brindar ayudas y soportes técnicos, pero aún falta entrar con mayor detalle en el desarrollo de tecnologías accesibles en sitios web y, sobre todo, que exista una entidad reguladora (cosa que no he se ha formalizado en otros países) que permita verificar que se está cumpliendo con las pautas de accesibilidad suministradas por la W3C.

La participación e integración de personas con discapacidad en el proceso de crear TIC accesibles es fundamental. Solamente una persona con algún tipo de discapacidad puede realmente especificar con claridad a qué tipo de situaciones se enfrenta, sobre todo para alcanzar las metas que requiere. El desarrollo de aplicaciones y sitios web accesibles está al alcance de la mano: las pautas de accesibilidad están ahí para analizarse y usarse; experimentar y desarrollar soportes técnicos que den oportunidad e igualdades para las personas que tengan algún tipo de discapacidad, independientemente del origen de esta.

Las universidades de nuestro país deben facilitar el espacio al diálogo para acoger e instruir procesos orientados al desarrollo de personas con discapacidad en el área de las TIC. De tal manera, que como lo han trabajado algunas universidades estatales del país, se sigan realizando aportes importantes en la educación, relacionados con este tema. Y se subsane, en algunas áreas, el desconocimiento existente respecto a la accesibilidad desde los diferentes ámbitos conversados, barriendo con los mitos.

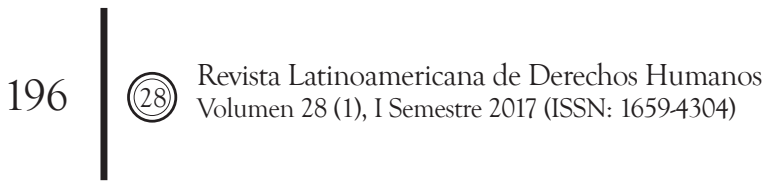


Resulta fundamental la integración de las empresas, del gobierno y del sector privado en el proceso, tanto para desarrollar mejores condiciones en las personas con discapacidad de forma laboral, como para su integración en el uso de herramientas accesibles. Se requiere invertir en proyectos de bien social que fomenten el desarrollo tecnológico en esta área, y creer en el retorno de la inversión de construir un bien social para una Costa Rica impulsadora de la accesibilidad universal.

\section{Referencias}

AFP. (2015) Accesibilidad física y cibernética para ciegos gana espacio en agenda latinoamericana. Recuperado de http://www.nacion. com/tecnologia/web/Accesibilidad-cibernetica-espacio-agendalatinoamericana_0_1519248152.html.

AGESIC. (s. f.) Capítulo III. Accesibilidad Web. Recuperado de http://agesic.gub. uy/innovaportal/file/549/1/Capitulo_3_Accesibilidad_v1_0.pdf

ASAMBLEA LEGISLATIVA DE LA REPUBLICA DE COSTA RICA. (1996). Ley N. ${ }^{\circ}$ 7600. Igualdad De Oportunidades Para Las Personas Con Discapacidad. Costa Rica: Autor.

Carreño, I. (2016). Colombia, Costa Rica y Perú a través de sus políticas de accesibilidad. Recuperado de http://www.mediatelecom.com. $\mathrm{mx} / \sim$ mediacom/index.php/agencia-informativa/noticias/item/125248colombia,-costa-rica-y-perú-a-través-de-sus-políticas-de-accesibilidad.

CEPAL. (2016). Tecnologías de información y comunicación para la inclusión y el empoderamiento de las personas con discapacidad en América Latina y el Caribe. Presentación PowerPoint. Diapositivas 14 - 18.

Chacón, K. (2015). FONATEL aspira a conectividad total de Costa Rica en el 2017. Recuperado de http://www.elfinancierocr.com/tecnologia/Fonatelconectividad-aldea_digital-San_Carlos-Internet_0_813518652.html

Chacón, K. (2016). ¿Es posible la tecnología accesible para todos los estratos sociales? Recuperado de http://www.elfinancierocr.com/tecnologia/ Tecnologia-accesible-estratos-sociales_0_899910001.html

FONATEL. (2016). Empoderar para el desarrollo: Proyecto de servicio universal más ambicioso (100\% territorios). Presentación PowerPoint. Diapositivas 5 - 6.

INEC. (2011). Población con discapacidad. Recuperado de http://www.inec.go.cr/ social/poblacion-con-discapacidad 
Ministerio de Salud (MINSA). (2004). La discapacidad en Costa Rica: Situación actual y perspectivas. Recuperado de http://www.bvs.sa.cr/php/situacion/ discapacidad.pdf

Mitchell, J. (s. f.). What Programming Language Is Primarily Used on the Web?. Recuperado de http://smallbusiness.chron.com/programming-languageprimarily-used-web-26635.html

Molina, K. y Cuevas, F. (2014). TIC y educación de personas con discapacidad en Costa Rica. Recuperado de www.oei.es/historico/congreso2014/memoriactei/1202.pdf

Mora, K. (2016). Traductor digital de LESCO avanza y alcanza la inclusión de 1284 glosas Recuperado de https://www.tec.ac.cr/hoyeneltec/2016/07/26/ traductor-digital-lesco-avanza-alcanza-inclusion-1284-glosas

Programa Sociedad de la Información y el conocimiento (PROSIC) - Universidad de Costa Rica. (UCR) (2011). TIC y personas con discapacidad en Costa Rica. Capítulo 9. Recuperado de http://www.prosic.ucr.ac.cr/sites/default/ files/recursos/informe_2011_cap9.pdf

RAE. (2016). Definición de concientizar - concienciar. Recuperado de 2017 de http://dle.rae.es/?id=A8mrPcH

RAE.(2016).Definición de sensibilizar. Recuperadodehttp://dle.rae.es/?id=XaGpWYI

Roig, Rafael de Asís. (2013). Sobre la accesibilidad universal. Recuperado de www. tiempodelosderechos.es/es/biblioteca/.../55-sobre-la-accesibilidad-universal.html.

SUTEL. (s. f.). Reglamento de Acceso Universal, Servicio Universal y Solidaridad. Recuperado de https://sutel.go.cr/sites/default/files/normativas/ reglamento_de_acceso_universal_servicio_universal_y_solidaridad.pdf

Universidad de la Rioja, DIALNET. (s. f.) Accesibilidad Web. Recuperado de https://dialnet.unirioja.es/info/accesibilidad

Vargas, M. (2016). Población sorda de Costa Rica tendrá canal Lesco en la web. Recuperado de http://www.nacion.com/tecnologia/apps/Poblacion-CostaRica-canal-Lesco_0_1580041996.html

W3C. (2008). Pautas de accesibilidad para el contenido web (WCAG) 2.0. Recuperado de http://www.sidar.org/traducciones/wcag20/es/

Recibido: 20/2/2017

Aceptado: 5/6/2017

198 (28) Revista Latinoamericana de Derechos Humanos 